\title{
Acurácia da determinação da idade gestacional no Sistema de Informações sobre Nascidos Vivos (SINASC): um estudo de base populacional
}

\section{Accuracy of gestational age assessment in Brazilian Information System on Live Birth (SINASC): a population study}

Precisión en la determinación de la edad gestacional en el Sistema de Información sobre Nacidos Vivos (SINASC): un estudio de base poblacional
Licia Barbosa Henriques 1

Erika Barreto Alves 2

Fernanda Morena dos Santos Barbeiro Vieira 2

Bruno Baptista Cardoso 2

Adriana Cristina Rodrigues D’Angeles 2

Oswaldo Gonçalves Cruz 2,3

Monique Felix Ribeiro da Silva 2

Valeria Saraceni 2

doi: 10.1590/0102-311X00098918

\section{Correspondência}

F. M. S. B. Vieira

Coordenação de Análise e Situação de Saúde, Secretaria

Municipal de Saúde do Rio de Janeiro.

Rua Afonso Cavalcanti 455, Rio de Janeiro, RJ

20211-110, Brasil.

nurse_fe@hotmail.com

1 Faculdade Vale do Cricaré, São Mateus, Brasil.

2 Secretaria Municipal de Saúde do Rio de Janeiro, Rio de Janeiro, Brasil.

3 Programa de Computação Científica, Fundação Oswaldo Cruz, Rio de Janeiro, Brasil. cional com dados do SINASC disponíveis no Departamento de Informática do SUS entre 2011 e 2015. As definições de prematuridade, baixo peso e asfixia ao nascer foram aquelas determinadas na literatura. A adequação do peso ao nascer com a idade gestacional foi calculada baseando-se nas curvas de Fenton e Intergrowth-21. Compararam-se as médias de peso pela presença ou não de prematuridade. A estimação da idade gestacional foi realizada pela DUM em 58,5\%, e 41,5\% utilizaram outro método. Encontrou-se que a proporção de prematuridade foi de $12 \%$ no grupo DUM e 8,4\% no grupo outro método, já o baixo peso ao nascer foi de 6,5\% e 8,4\%, respectivamente. A média de peso dos prematuros no grupo DUM foi maior. O uso da DUM como estimador da idade gestacional superestimou a proporção de peso maior ou igual a $2.500 \mathrm{~g}$ nos prematuros, o que não parece compatível com a distribuição esperada para esta faixa. A DUM favoreceu a "correção" da prematuridade para os parâmetros comparáveis aos de estudos com dados primários, embora as distorções encontradas entre idade gestacional e peso ao nascer possam indicar que ainda existem problemas com este estimador.

Sistemas de Informação; Declaração de Nascimento; Idade Gestacional; Recém-Nascido Prematuro; Saúde da Criança 


\section{Introdução}

A partir de 1990, quando o Ministério da Saúde implantou o Sistema de Informações sobre Nascidos Vivos (SINASC) por meio da Declaração de Nascido Vivo (DNV), passou a ser possível conhecer e acompanhar as variáveis relativas à gravidez, ao parto e ao nascimento (por exemplo: peso, idade gestacional, Apgar) de maneira sistemática, favorecendo a criação e a redefinição de políticas de saúde 1 .

O nascimento pré-termo é definido como aquele que resulta em um nascido vivo com idade gestacional inferior a 37 semanas 2 . A prematuridade é motivo de grande preocupação, visto que suas complicações estão relacionadas com a mortalidade infantil, em especial a mortalidade neonatal. Os nascimentos prematuros geram uma demanda adicional sobre os serviços de saúde pela maior complexidade, muitas vezes em unidades de tratamento intensivo, podendo ter repercussões na saúde em longo prazo ${ }^{3}$.

Não há um método único para a medida da idade gestacional. Métodos estimadores (exame físico, data da última menstruação, ultrassonografia) são usualmente utilizados, apresentando precisão e limitações variáveis 3 . A data da última menstruação (DUM) tem sido considerada como a primeira escolha, sendo dependente da memória da mulher grávida 4 e com o risco de não ser a correta, mesmo quando lembrada, por conta de ciclos menstruais irregulares e ocorrência de sangramento no primeiro trimestre da gestação 5 .

Uma crescente indicação da ultrassonografia (USG) para estimar a data provável do parto e a idade gestacional do recém-nascido tem sido observada nos últimos anos. Entretanto, a determinação da idade gestacional pela USG depende de medidas fetais estabelecidas com base na idade gestacional determinada pela DUM e pode variar dependendo da população ${ }^{6}$. Além disso, em alguns locais, o elevado custo tem sido uma dificuldade encontrada na sua utilização em grande escala, principalmente em países que se encontram em processo de desenvolvimento. Nas situações em que a qualidade da informação da DUM seja questionável e a mãe não tenha realizado a USG no primeiro trimestre de gestação, a idade gestacional pode ser estimada pela observação das características físicas e neurológicas do recém-nascido nos seus primeiros dias de vida. Porém, a utilização do exame clínico do recém-nascido para tal estimativa também é alvo de críticas, pois existe uma grande inadequação na maioria dos escores estabelecidos para esta avaliação 5 .

A prevalência de nascimento pré-termo tem apresentado uma tendência crescente em vários países, inclusive naqueles desenvolvidos. A Organização Mundial da Saúde 7 estima que ocorram, anualmente, 15 milhões de nascimentos pré-termo, mais de 10\% do total dos nascimentos. Dados da pesquisa Nascer no Brasil, estudo de base populacional, mostraram uma prevalência de 11,5\% de prematuros, associados principalmente aos partos cesáreos 8 . Segundo Silveira et al. 9, a proporção de prematuridade no Brasil cresceu entre 1994 e 2005, acompanhada de uma melhoria no preenchimento da variável idade gestacional no início do período. A correção da prematuridade encontrada no SINASC por estimativas baseadas em dados primários de estudos brasileiros concluiu pela subestimação da prematuridade, com dados observados de 6 a $7 \%$ entre 2000 e 2011, quando eram esperados de 11 a $12 \% 10$.

Em 2011, a DNV passou a incluir a variável DUM para o cálculo automático da idade gestacional no SINASC e disponibilização de modo desagregado. Quando não é possível informar a DUM (padrão-ouro), a idade gestacional é informada em número de semanas e é apontado o uso de "outro método" estimador, seja exame físico ou ultrassonografia. A resposta "ignorado" também é aceita pelo sistema, tanto para o método estimador quanto para a idade gestacional. Quando estão disponíveis na DNV as informações da DUM e a idade gestacional estimada por outro método, existe a preferência pela DUM, selecionada exclusivamente pelo sistema, para o cálculo automático da idade gestacional 11 .

Utilizando os dados do SINASC disponíveis no TabNet (http://www2.datasus.gov.br/DATASUS/ index.php?area=0205\&id=6936), verificou-se que o baixo peso ao nascer $(<2.500 \mathrm{~g})$ permaneceu estável no Brasil entre 2006 e 2015, e a prematuridade aumentou bruscamente, de 7,1\% em 2010 para 9,8\% em 2011, ano da mudança da DNV (Figura 1).

O objetivo deste estudo foi comparar, no SINASC, a idade gestacional gerada pela DUM com a de outros estimadores e correlacioná-la com o peso ao nascer, no período de 2011-2015, a partir da mudança do modelo da DNV, para avaliar a acurácia da determinação da idade gestacional em uma base populacional. 


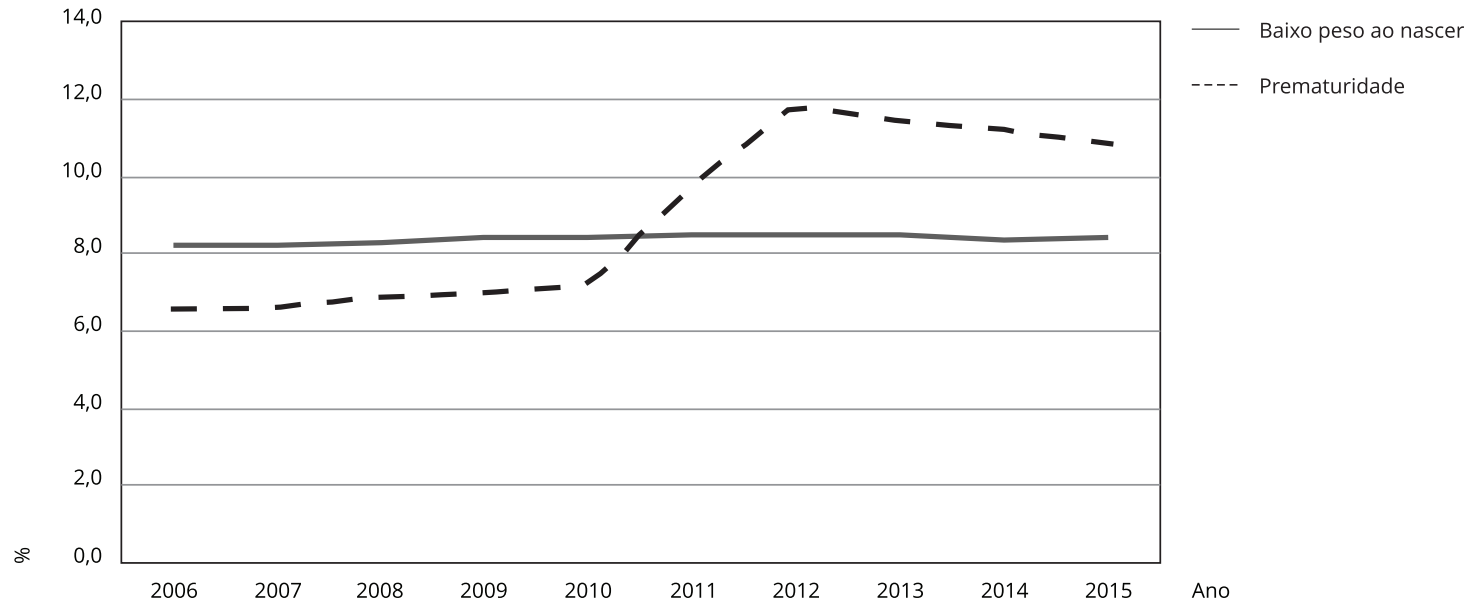

\section{Métodos}

Foi realizado um estudo transversal da base populacional do SINASC, disponível no Departamento de Informática do SUS (DATASUS) em livre acesso, para o período de 2011-2015. Ter a DUM ou outro estimador decorreu do preenchimento da DNV na maternidade, não sendo possível alocar mais de uma metodologia para cada caso, pois a DNV só admite a DUM. A distribuição foi considerada aleatória e não diferencial para todos os nascimentos vivos no Brasil entre 2011 e 2015, uma vez que o Manual de Instruções para o Preenchimento da Declaração de Nascido Vivo 11 dá preferência à DUM e esclarece quais outros métodos podem ser usados, se a DUM não estiver disponível.

Os dados de peso ao nascer e idade gestacional, avaliados pela DUM ou outro método estimador, foram tabulados retirando-se os casos sem informação de idade gestacional nas duas variáveis (gestacao e semagestac) ou com a idade gestacional menor do que 19 semanas, os casos sem peso ao nascer informado e os extremos de peso $(<500 \mathrm{~g}$ ou $>4.500 \mathrm{~g})$, além dos produtos decorrentes de gestações múltiplas 12. A inclusão dessas últimas poderia enviesar os resultados por serem reconhecidamente associadas com prematuridade e baixo peso ao nascer.

A prematuridade foi definida como a idade gestacional abaixo de 37 semanas e o baixo peso ao nascer aquele abaixo de $2.500 \mathrm{~g}$. A adequação do peso ao nascer com a idade gestacional foi calculada com base nas curvas de Fenton 13 e Intergrowth-21 14, classificando-se como pequenos para a idade gestacional (PIG) aqueles situados abaixo do percentil 3 e grandes para a idade gestacional (GIG) aqueles acima do percentil 97 nas curvas. A asfixia ao nascer foi definida como o Apgar de 50 minuto abaixo de 7.

Foram comparadas as médias de peso ao nascer pela presença ou não de prematuridade (idade gestacional < 37 semanas) pelo teste $t$ de Student. As variáveis categóricas apresentadas na Tabela 1 foram comparadas para homogeneidade de proporções pelo teste do qui-quadrado de Pearson e as variáveis contínuas da mesma tabela utilizaram o teste t ou o teste de Wilcoxon, de acordo com a distribuição normal ou não, verificada pelo teste de Kolmogorov-Smirnoff.

As análises foram feitas nos pacotes estatísticos Stata versão 11.0 (https://www.stata.com) e R (http://www.r-project.org). 


\section{Resultados}

Entre 2011 e 2015 foram informados 14.719 .903 nascidos vivos no Brasil. Nesta análise foram considerados 13.782 .517 registros, após as exclusões mencionadas, que representaram 6,3\% do total de nascidos vivos.

As características maternas, da gestação, parto e nascimento entre 2011 e 2015, divididas pelo método estimador da idade de gestacional, estão na Tabela 1. A estimação da idade gestacional foi realizada pela DUM em 58,5\%, e 41,5\% usaram outro método (exame físico ou outro). As mães cuja idade gestacional foi estimada pela DUM apresentaram uma maior proporção de idade $<20$ anos $(19,5 \%$ versus $18,4 \%)$ e de escolaridade < 4 anos (4,9\% versus 3,8\%), e uma menor proporção de viver sem companheiro (39,3\% versus $50,6 \%)$ e de cor branca (33,4\% versus $44,1 \%)$, quando comparadas às com idade gestacional estimada por outro método. A proporção de prematuridade (idade gestacional $<37$ semanas) foi de $12 \%$ no grupo DUM e de 8,4\% no grupo outro método, e o baixo peso ao nascer (peso $<2.500 \mathrm{~g}$ ) foi de $6,5 \%$ e $8,4 \%$, respectivamente. O percentual de asfixia ao nascer foi de $1 \%$ no grupo DUM e 1,1\% no grupo outro método. A mediana de número de consultas no pré-natal foi igual a 7 no grupo DUM e 8 no outro método. Para todas as variáveis a comparação dos grupos resultou significativa. Era esperado que, dado o tamanho da população do estudo, qualquer pequena diferença na comparação das variáveis resultasse em diferença significativa. A maior proporção de ignorados na variável raça/cor materna foi registrada pela implantação gradativa do novo formulário da DNV em 2011, quando a variável foi introduzida ao mesmo tempo que a DUM na variável idade gestacional.

As médias de peso dos nascidos vivos entre 2011 e 2015 estratificadas por idade gestacional e método estimador estão na Tabela 2, na qual é possível verificar uma mínima variação da média do

\section{Tabela 1}

Características sociodemográficas maternas, da gestação, do parto e nascimento por método estimador da idade gestacional. Brasil, 2011-2015.

\begin{tabular}{|c|c|c|c|}
\hline \multirow[t]{3}{*}{ Características } & \multicolumn{2}{|c|}{ Método } & \multirow[t]{3}{*}{ Valor de $p$} \\
\hline & DUM & Outro & \\
\hline & {$[n=8.056 .457]$} & {$[n=5.726 .060]$} & \\
\hline Idade materna [mediana, IIQ] & $25(21-31)$ & $26(21-31)$ & $<0,001$ \\
\hline \multicolumn{4}{|c|}{ Faixa etária materna (anos) [n (\%)] } \\
\hline$<20$ & $1.572 .115(19,5)$ & $1.054 .136(18,4)$ & \\
\hline $20-29$ & $4.070 .425(50,5)$ & $2.840 .713(49,6)$ & \\
\hline $30-39$ & $2.232 .957(27,7)$ & $1.683 .129(29,4)$ & \\
\hline 40 e mais & $180.847(2,3)$ & $147.945(2,6)$ & \\
\hline Ignorado & $113(0,0)$ & $137(0,0)$ & $<0,001$ \\
\hline \multicolumn{4}{|l|}{ Cor da mãe [n (\%)] } \\
\hline Branca & $2.577 .670(32,0)$ & $1.874 .080(32,7)$ & \\
\hline Preta & $380.160(4,7)$ & $278.068(4,9)$ & \\
\hline Amarela & $29.366(0,4)$ & $17.935(0,3)$ & \\
\hline Parda & $4.664 .485(57,9)$ & $2.057 .079(35,9)$ & \\
\hline Indígena & $67.093(0,8)$ & $22.431(0,4)$ & \\
\hline Ignorada & $337.683(4,2)$ & $1.476 .467(25,8)$ & $<0,001$ \\
\hline \multicolumn{4}{|c|}{ Escolaridade materna (anos) [n (\%)] } \\
\hline Nenhuma & $56.849(0,7)$ & $36.003(0,6)$ & \\
\hline $1-3$ & $335.771(4,2)$ & $182.838(3,2)$ & \\
\hline $4-7$ & $1.772 .463(22,0)$ & $1.164 .448(20,3)$ & \\
\hline $8-11$ & $4.491 .930(55,8)$ & $3.227 .489(56,4)$ & \\
\hline 12 e mais & $1.252 .749(15,6)$ & $1.051 .071(18,4)$ & \\
\hline Ignorada & $146.695(1,7)$ & $64.211(1,1)$ & $<0,001$ \\
\hline
\end{tabular}


Tabela 1 (continuação)

\begin{tabular}{|c|c|c|c|}
\hline \multirow[t]{3}{*}{ Características } & \multicolumn{2}{|c|}{ Método } & \multirow[t]{3}{*}{ Valor de $p$} \\
\hline & DUM & Outro & \\
\hline & {$[n=8.056 .457]$} & {$[n=5.726 .060]$} & \\
\hline \multicolumn{4}{|l|}{ Estado civil da mãe [n (\%)] } \\
\hline Solteira & 3.039.792 $(37,7)$ & $2.791 .486(48,8)$ & \\
\hline Casada & $2.652 .677(32,9)$ & $1.885 .451(32,9)$ & \\
\hline Viúva & $14.957(0,2)$ & $11.127(0,2)$ & \\
\hline Separada & $73.841(0,9)$ & $70.420(1,2)$ & \\
\hline União estável & $2.173 .059(27,0)$ & $916.450(16,0)$ & \\
\hline Ignorado & $102.131(1,3)$ & $51.126(0,9)$ & $<0,001$ \\
\hline \multicolumn{4}{|l|}{ Duração da gestação (semanas) [n (\%)] } \\
\hline$<22$ & $2.779(0,03)$ & $1.791(0,03)$ & \\
\hline $22-27$ & $30.6119(0,4)$ & $25.183(0,4)$ & \\
\hline $28-31$ & $77.920(1,0)$ & $50.268(0,9)$ & \\
\hline $32-36$ & $858.208(10,6)$ & $402.945(7,0)$ & \\
\hline $37-41$ & $6.640 .871(82,4)$ & $5.214 .210(91,1)$ & \\
\hline$\geq 42$ & $31.663(0,6)$ & $31.663(0,6)$ & $<0,001$ \\
\hline \multicolumn{4}{|l|}{ Tipo de parto [n (\%)] } \\
\hline Vaginal & $3.605 .703(44,8)$ & $2.520 .101 .(44,0)$ & \\
\hline Cesáreo & 3.202.434 (55,2) & $3.202 .434(56,0)$ & \\
\hline Peso ao nascer [média, DP] & $3.212(503)$ & $3.167(534)$ & $<0,001$ \\
\hline \multicolumn{4}{|l|}{ Peso ao nascer (g) [n (\%)] } \\
\hline $500-999$ & $24.364(0,3)$ & $30.232(0,5)$ & \\
\hline $1.000-1.499$ & $40.404(0,5)$ & $44.920(0,8)$ & \\
\hline $1.500-1.999$ & $88.467(1,1)$ & $87.812(1,5)$ & \\
\hline $2.000-2.499$ & $372.666(4,6)$ & $315.375(5,5)$ & \\
\hline $2.500-2.999$ & $1.820 .966(22,6)$ & $1.368 .567(23,9)$ & \\
\hline $3.000-3.499$ & $3.463 .060(43,0)$ & $2.415 .343(42,2)$ & \\
\hline $3.500-3.999$ & $1.858 .940(23,1)$ & $1.222 .830(21,4)$ & \\
\hline $4.000-4.500$ & $387.320(4,8)$ & $240.981(4,2)$ & $<0,001$ \\
\hline \multicolumn{4}{|l|}{ Asfixia ao nascer [n (\%)] } \\
\hline $\operatorname{Sim}$ & $80.450(1,0)$ & $64.632(1,1)$ & \\
\hline Não & $7.710 .306(99,0)$ & $5.571 .047(98,9)$ & $<0,001$ \\
\hline \multicolumn{4}{|l|}{ Número de consultas de pré-natal [n (\%)] } \\
\hline Nenhuma & $96.785(1,2)$ & $152.310(2,7)$ & \\
\hline $1-3$ & $534.152(6,7)$ & $402.354(7,1)$ & \\
\hline $4-6$ & $2.209 .927(27,5)$ & $1.399 .268(24,7)$ & \\
\hline 7 e mais & $5.189 .366(64,6)$ & $3.701 .856(65,5)$ & \\
\hline Ignorado & $26.227(0,3)$ & $70.272(1,2)$ & $<0,001$ \\
\hline Número de consultas pré-natal [mediana, IIQ] & $7(6-9)$ & $8(6-10)$ & $<0,001$ \\
\hline
\end{tabular}

DP: desvio padrão; DUM: data da última menstruação; IIQ: intervalo interquartil.

peso por cada estimador, distribuído por prematuro precoce ( $<32$ semanas), prematuro tardio (32-36 semanas) e a termo ( $\geq 37$ semanas). Entre todos os prematuros, a média de peso foi estatisticamente maior no grupo da DUM, quando comparada com o outro método, em todos os anos examinados, com uma diferença média de 493g. A diferença na média de peso entre os dois grupos de estimadores ocorreu tanto nos prematuros precoces como nos tardios. Já entre os nascidos vivos de gestação a termo a diferença entre as médias de peso da DUM e do outro método foi de apenas $23 \mathrm{~g}$.

O peso médio dos prematuros nascidos em 2010 foi de $2.305 \mathrm{~g}$ e no período de 2011-2015, de 2.612 g. Entre os nascidos a termo, a média foi de $3.257 \mathrm{~g}$ em 2010 e de 3.261 no período do estudo. 
Tabela 2

Média do peso ao nascer em gramas por idade gestacional e método estimador da idade gestacional. Brasil, 2011-2015.

\begin{tabular}{|c|c|c|c|c|c|c|}
\hline \multirow[t]{3}{*}{ Ano } & \multicolumn{6}{|c|}{ Idade gestacional (semanas) } \\
\hline & \multicolumn{2}{|c|}{$<32$} & \multicolumn{2}{|c|}{$32-36$} & \multicolumn{2}{|c|}{$\geq 37$} \\
\hline & DUM & Outro & DUM & Outro & DUM & Outro \\
\hline 2011 & 2.096 & 1.328 & 2.887 & 2.475 & 3.271 & 3.237 \\
\hline 2012 & 1.953 & 1.304 & 2.890 & 2.484 & 3.270 & 3.245 \\
\hline 2013 & 1.992 & 1.299 & 2.882 & 2.472 & 3.268 & 3.246 \\
\hline 2014 & 1.950 & 1.264 & 2.880 & 2.475 & 3.274 & 3.254 \\
\hline 2015 & 1.983 & 1.276 & 2.866 & 2.474 & 3.275 & 3.258 \\
\hline
\end{tabular}

DUM: data da última menstruação.

Fonte: Departamento de Informática do SUS. Sistema de Informações sobre Nascidos Vivos (http://www2.datasus.gov. br/DATASUS/index.php?area=060702).

Novamente, a diferença nas médias de peso foi maior entre os prematuros, quando se comparou o ano imediatamente anterior ao da mudança do SINASC (2010) com o período analisado.

A Tabela 3 mostra uma maior proporção de prematuros com peso normal entre os avaliados pela $\operatorname{DUM}(9,1 \%)$ do que nos avaliados por outro método (3,8\%). A adequação do peso ao nascer pela idade gestacional mostrou uma maior proporção de GIG entre os avaliados pela DUM $(6,8 \%)$ do que por outro método (3,9\%) (dados não apresentados).

A Figura 2 apresenta os boxplots da distribuição do peso ao nascer por semanas de gestação, estratificados pelo método estimador, DUM ou outro. A mediana de peso é mais alta entre aqueles nascidos com 32-37 semanas de idade gestacional estimadas pela DUM, com tendência de aproximação ao outro método conforme aumenta a idade gestacional, mantendo-se semelhante entre a 38 a e a 45 a semanas.

\section{Discussão}

Este estudo de base populacional mostrou que a utilização da DUM como método estimador da idade gestacional trouxe um aumento na média de peso ao nascer dos nascidos vivos prematuros, o que não ocorreu com os prematuros com idade gestacional estimada por outro método, ao mesmo tempo em que se identificou um aumento da prematuridade no SINASC, sem modificação do baixo peso ao nascer no período.

O Ministério da Saúde atribuiu, no documento Consolidação do Sistema de Informações sobre Nascidos Vivos - 2011 15, o aumento da prematuridade verificada no SINASC a partir de 2011 a um ajuste na qualidade da informação. O esperado aumento da prematuridade que se pretendia corrigir com a mudança na DNV em 201110 ocorreu, principalmente, no grupo de prematuros tardios (idade gestacional de 32-36 semanas), embora tenha afetado, também, os prematuros precoces (idade gestacional < 32 semanas), conforme demonstrado na presente avaliação. Outro estudo que comparou a DUM com a ultrassonografia em gestações únicas nos Estados Unidos evidenciou um aumento da proporção de prematuros com o cálculo pela $\operatorname{DUM}(8,7 \%$ versus 7,9\%), com maior proporção de peso normal entre 32 e 36 semanas de idade gestacional. Tanto a sensibilidade como a especificidade da DUM resultaram baixas, quando comparadas à ultrassonografia como padrão-ouro 16 .

O aumento da média de peso dos prematuros não foi acompanhado de alteração da média de peso dos nascidos a termo entre os com idade gestacional estimada pela DUM. A melhor identificação dos prematuros tardios entre 32 e 36 semanas de idade gestacional pela DUM poderia justificar esse achado. Como o percentual de nascidos vivos a termo (88\%) é muito superior ao de prematuros (12,0\%), a média de peso não apresentou alteração no grupo a termo. A análise das médias de peso apresentada na Tabela 2 mostra que, para o grupo com idade gestacional menor que 32 semanas, a média de peso é, no mínimo e para todos os anos avaliados, 50\% maior para o grupo estimado pela DUM. Sendo esse 
Tabela 3

Distribuição dos nascidos vivos por idade gestacional, peso ao nascer e método estimador da idade gestacional. Brasil, 2011-2015.

\begin{tabular}{lcc} 
Peso ao nascer/Idade gestacional (semanas) & \multicolumn{2}{c}{ Método estimador [n (\%)] } \\
& DUM & \\
\hline $\begin{array}{l}\text { Peso }<2.500 \mathrm{~g} \\
\quad 32\end{array}$ & $71.846(13,7)$ & $72.327(15,1)$ \\
$32-36$ & $208.321(39,6)$ & $208.030(43,5)$ \\
$\geq 37$ & $246.004(46,7)$ & $197.982(41,4)$ \\
Peso $\geq 2.500$ & & \\
$<32$ & $39.472(0,5)$ & $4.915(0,1)$ \\
$32-36$ & $649.887(8,6)$ & $194.915(3,7)$ \\
$\geq 37$ & $6.840 .927(90,9)$ & $5.047 .891(96,2)$
\end{tabular}

DUM: data da última menstruação.

Fonte: Departamento de Informática do SUS. Sistema de Informações sobre Nascidos Vivos (http://www2.datasus.gov. br/DATASUS/index.php?area=060702).

Figura 2

Distribuição do peso ao nascer por semanas de gestação, estratificado pelo método estimador. Brasil, 2010-2015.

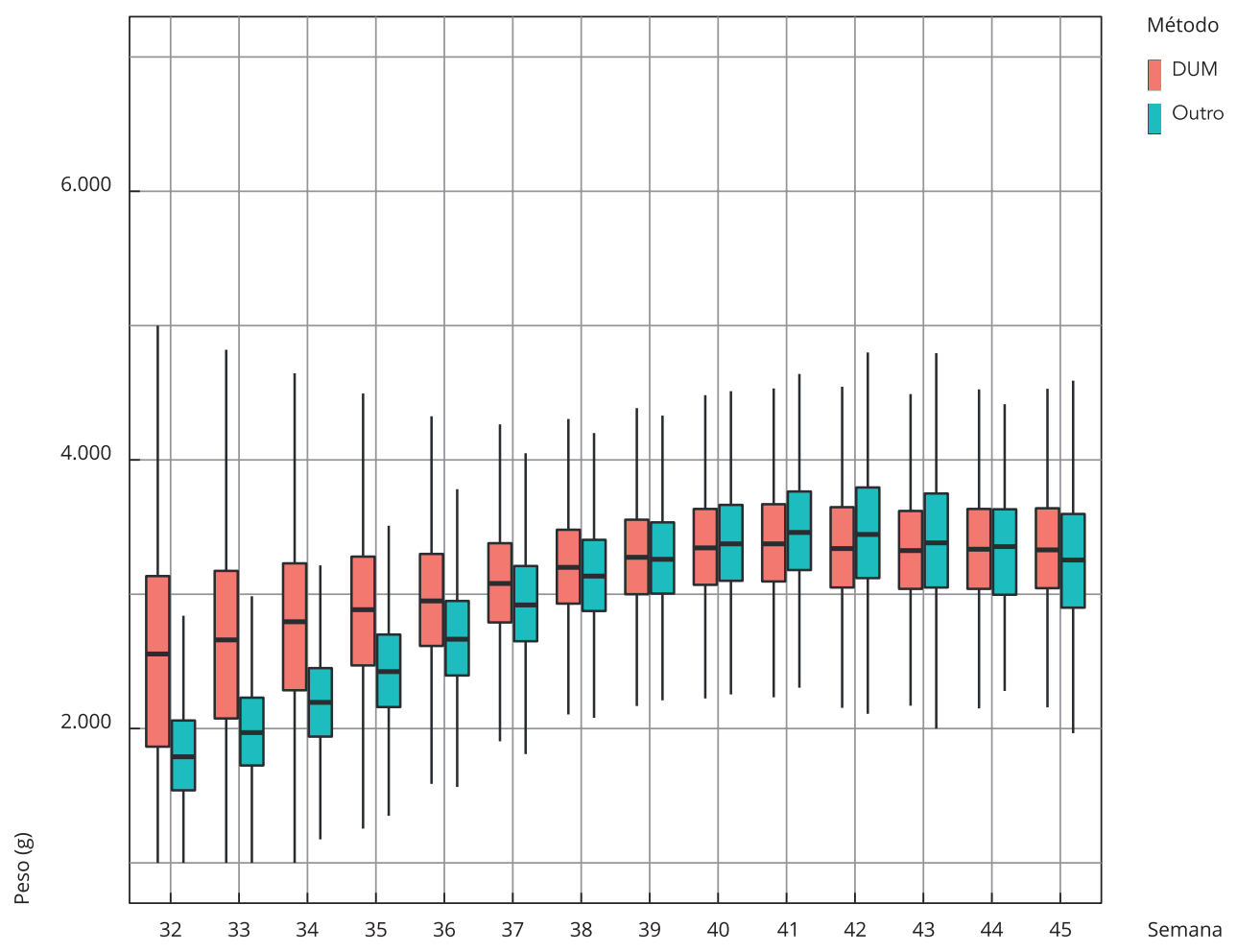

DUM: data da última menstruação. 
grupo o de maior risco, a estimação da idade gestacional pela DUM desvia para a prematuridade precoce um grande número de nascidos vivos com peso $>2.500 \mathrm{~g}$, inviabilizando a DUM para o cálculo acurado da idade gestacional. A estimação da idade gestacional pela DUM trouxe um aumento do percentual de prematuros com peso normal $(\geq 2.500 \mathrm{~g})$ entre os com idade gestacional estimada por este método (9,1\%), quando comparados aos estimados por outro método $(3,8 \%)$, o que não era de se esperar de um melhor estimador. Em uma coorte de nascidos vivos americanos, o mesmo aumento de peso nos prematuros foi demonstrado, com os autores sugerindo revisitar a DUM como método estimador nos Estados Unidos 17. Com dados primários de nascimentos em duas cidades do Estado do Rio de Janeiro, Pereira et al. 18 demonstraram que a proporção de prematuridade variou de 9,4\% pelo método de Capurro, subestimando a proporção de prematuridade, a 20,9\% com a DUM, claramente superestimando os prematuros.

A curva de distribuição de peso por idade gestacional e por estimador do presente trabalho evidenciou que o peso nos nascidos vivos cuja idade gestacional foi estimada pela DUM ficou sempre acima do peso dos que utilizaram outro método, principalmente nas faixas de $<32$ semanas e de 32-36 semanas. Um estudo que avaliou o peso ao nascer por idade gestacional calculada pela DUM mostrou 6\% de outliers na distribuição do peso 14 . Um trabalho nacional mostrou que a DUM também sobre-enumerava a pós-maturidade 17 , o que não foi visto aqui, em que as medianas de peso para idade gestacional de 43-45 semanas foram bastante semelhantes nos dois grupos.

A alteração da DNV em 2011 já trouxe um pequeno incremento na prematuridade, como pode ser visto no grupo "outro método" com 8,4\%, quando a proporção de prematuridade até 2010 era cerca de $7 \%$, provavelmente relacionada ao fato de ser necessário preencher o número de semanas e não mais marcar uma categoria de idade gestacional. Por outro lado, a mudança da forma de coleta do dado não afetou de maneira abrupta a variável "número de consultas de pré-natal”, que já vinha apresentando tendência de aumento para "7 e mais consultas". O mesmo aconteceu com a variável "idade da mãe", agora derivada da data de nascimento, em aumento gradativo da faixa de 30-39 anos, acompanhada da diminuição entre 15 e 25 anos (dados disponíveis no TabNet, http://www2.datasus.gov.br/ DATASUS/index.php?area=02).

Entre as limitações deste trabalho está o fato de o SINASC não discriminar o "outro método" usado para a estimação da idade gestacional, não sendo possível ter a anotação clara da utilização de ultrassonografia e de quando esta foi realizada. Somente os trabalhos com dados primários servem de fonte para a comparação da utilização da USG com a DUM e o exame do recém-nascido. A pesquisa Nascer no Brasil sugere cautela no uso da DUM para a estimação da idade gestacional, favorecendo o uso da USG precoce, realizada entre 7 e 20 semanas de gestação ${ }^{19}$. No Reino Unido, a USG precoce é a recomendação para a avaliação da idade gestacional 20. No Brasil, a USG também pode ser oferecida, dada a incorreções apresentadas pela estimação pela DUM 21

Ao avaliar-se a base populacional, excluídas as gestações múltiplas, verificou-se um excesso nascidos vivos prematuros com peso não compatível com a idade gestacional, quando a idade gestacional foi estimada pela DUM, reforçando não ser este o melhor estimador no Brasil. Apesar de se tratar de dados secundários, usar o SINASC como fonte de dados no país é um potente recurso para estudos epidemiológicos 22,23 e pode ajudar a qualificar estratégias que visam a melhorar os dados sobre a saúde da população e a formulação de políticas públicas.

\section{Conclusões}

A utilização da DUM como método estimador prioritário da idade gestacional no SINASC a princípio corrigiu a subnotificação de nascimentos prematuros no país para níveis compatíveis com as estimativas baseadas em estudos com dados primários. Este estudo mostra, no entanto, que a média de peso do total de nascimentos prematuros passou a ser maior que $2.500 \mathrm{~g}$, principalmente devido aos prematuros tardios estimados pela DUM. As distorções entre peso e idade gestacional foram mais frequentes entre os nascimentos com idade gestacional estimada pela DUM, tanto entre os prematuros quanto nos nascimentos a termo. Os resultados deste trabalho evidenciaram que o método não tem a precisão esperada e elevou a proporção de prematuridade no SINASC pela classificação inadequada de nascimentos a termo. 


\section{Colaboradores}

L. B. Henriques, E. B. Alves, F. M. S. B. Vieira, B. B. Cardoso, A. C. R. D’Angeles, O. G. Cruz, M. F. R. Silva e V. Saraceni contribuíram substancialmente para a concepção, análise e interpretação dos dados; e aprovaram a versão final a ser publicada.

\section{Informações adicionais}

ORCID: Licia Barbosa Henriques (0000-00028524-2898); Erika Barreto Alves (0000-0001-79508947); Fernanda Morena dos Santos Barbeiro Vieira (0000-0002-2010-761X); Bruno Baptista Cardoso (0000-0001-8147-9452); Adriana Cristina Rodrigues D’Angeles (0000-0002-8540-4906); Oswaldo Gonçalves Cruz (0000-0002-3289-3195); Monique Felix Ribeiro da Silva (0000-0002-2309-5511); Valeria Saraceni (0000-0001-7360-6490).

\section{Referências}

1. Mello Jorge MHP, Gotlieb SLD, Soboll MLMS, Almeida MF, Latorre MRDO. Avaliação do sistema de informação sobre nascidos vivos e o uso de seus dados em epidemiologia e estatísticas de saúde. Rev Saúde Pública 1993; 27 Suppl:1-46.

2. WHO: recommended definitions, terminology and format for statistical tables related to the perinatal period and use of a new certificate for cause of perinatal deaths. Modifications recommended by FIGO as amended October 14, 1976. Acta Obstet Gynecol Scand 1977; 56:247-53.

3. Blencowe H, Lee AC, Cousens S, Bahalim A, Narwal R, Zhong N, et al. Preterm birth-associated neurodevelopmental impairment estimates at regional and global levels for 2010. Pediatr Res 2013; 74 Suppl 1:17-34.

4. Alexander GR, Allen MC. Conceptualization, measurement, and use of gestational age. I. Clinical and public health practice. J Perinatol 1996; 16:53-9.

5. Moraes CL, Reichenheim ME. Validade do exame clínico do recém-nascido para a estimação da idade gestacional: uma comparação do escore New Ballard com a data da última menstruação e ultra-sonografia. Cad Saúde Pública 2000; 16:83-94.

6. Lynch CD, Zhang J. The research implications of the selection of a gestational age estimation method. Paediatr Perinat Epidemiol 2007; 21Suppl 2:86-96.

7. Chawanpaiboon S, Vogel JP, Moller AB, Lumbiganon P, Petzold M, Hogan D, et al. Global, regional, and national estimates of levels of preterm birth in 2014: a systematic review and modelling analysis. Lancet Glob Health 2019; 7:e37-e46

8. Leal MC, Esteves-Pereira AP, Nakamura-Pereira M, Torres JA, Theme-Filha M, Domingues RMSM, et al. Prevalence and risk factors related to preterm birth in Brazil. Reprod Health 2016; 13 Suppl 3:127.

9. Silveira MF, Santos IS, Matijasevich A, Malta DC, Duarte EC. Nascimentos pré-termo no Brasil entre 1994 e 2005 conforme o Sistema de Informações sobre Nascidos Vivos (SINASC). Cad Saúde Pública 2009; 5:126775.

10. Matijasevich A, Silveira MF, Matos ACG, Rabello ND, Fernandes RM, Maranhão AG, et al. Estimativas corrigidas da prevalência de nascimentos pré-termo no Brasil, 2000 a 2011. Epidemiol Serv Saúde 2013; 22:557-64.

11. Departamento de Análise da Situação de Saúde, Secretaria de Vigilância em Saúde, Ministério da Saúde. Manual de instruções para o preenchimento da Declaração de Nascido Vivo. Brasília: Ministério da Saúde; 2011. (Série A Normas e Manuais Técnicos).

12. Kramer MS. Determinants of low birth weight: methodological assessment and meta-analysis. Bull World Health Organ 1987; 65:663-737. 
13. Fenton TR, Kim JH. A systematic review and meta-analysis to revise the Fenton growth chart for preterm infants. BMC Pediatr 2013; 13:59.

14. Villar J, Papageorghiou AT, Pang R, Ohuma EO, Ismail LC, Barros FC, et al. The likeness of fetal growth and newborn size across non-isolated populations in the INTERGROWTH-21 st Project: the Fetal Growth Longitudinal Study and Newborn CrossSectional Study. Lancet Diabetes Endocrinol 2014; 2:781-92.

15. Coordenação Geral de Informações e Análises Epidemiológicas, Secretaria de Vigilância em Saúde, Ministério da Saúde. Consolidação do Sistema de Informações sobre Nascidos Vivos - 2011. http://tabnet.datasus.gov.br/cgi/ sinasc/Consolida_Sinasc_2011.pdf (acessado em 15/Mai/2018).

16. Dietz PM, England LJ, Callaghan WM, Pearl M, Wier ML, Kharrazi M. A comparison of LMP-based and ultrasound-based estimates of gestational age using linked California livebirth and prenatal screening records. Paediatr Perinat Epidemiol 2007; 21 Suppl 2:62-71.

17. Callaghan WM, Dietz PM. Differences in birth weight for gestational age distributions according to the measures used to assign gestational age. Am J Epidemiol 2010; 171:826-36.

18. Pereira AP, Dias MA, Bastos MH, Gama SG, Leal MC. Determining gestational age for public health care users in Brazil: comparison of methods and algorithm creation. BMC Res Notes 2013; 6:60.
19. Pereira APE, Leal MC, Gama SGN, Domingues RMSM, Schilithz AOC, Bastos MH. Determinação da idade gestacional com base em informações do estudo Nascer no Brasil. Cad Saúde Pública 2014; 30 Suppl:S59-70.

20. National Institute for Health and Clinical Excellence. Antenatal care: routine care for the healthy pregnant woman. London: RCOG Press; 2008.

21. Departamento de Atenção Básica, Secretaria de Atenção à Saúde, Ministério da Saúde. Atenção ao pré-natal de baixo risco. Brasília: Editora do Ministério da Saúde; 2012. (Série A. Normas e Manuais Técnicos). (Cadernos de Atenção Básica, 32).

22. Costa CE, Gotlieb SLD. Estudo epidemiológico do peso ao nascer a partir da Declaração de Nascido Vivo. Rev Saúde Pública 1998; 32:328-34

23. Oliveira MM, Andrade SSCA, Dimech GS, Oliveira JCG, Malta DC, Rabello Neto DL, et al. Avaliação do Sistema de Informações sobre Nascidos Vivos. Brasil, 2006 a 2010. Epidemiol Serv Saúde 2015; 24:629-40. 


\section{Abstract}

The prevalence of preterm births has shown a growing trend in many countries, including developed ones. Studies in Brazil have shown that the Information System on Live Births (SINASC, in Portuguese), until 2010, underestimated the prevalence of preterm births, when compared with studies based on primary data. Starting in 2011, gestational age at birth has been calculated in SINASC according to the last menstrual period (LMP), when available. This study sought to evaluate the accuracy of the gestational age assessment using LMP, compared with two other estimates, and correlate it with birth weight. This is a population study with data from SINASC available from Brazilian Health Informatics Department between 2011 and 2015. Definitions of preterm birth, low birth weight and birth asphyxia were taken from the literature. Adequacy of birth weigh to gestational age was calculated based on Fenton and Intergrowth-21 curves. We compared weight means according to the presence or lack of preterm birth. gestational age assessment was based on LMP in $58.5 \%$ and $41.5 \%$ used another method. We found that the preterm proportion was $12 \%$ in the LMP group and $8.4 \%$ in the other method group, while low birth weight was $6.5 \%$ and $8.4 \%$, respectively. Mean weight of preterm infants was higher in the LMP group. Use of LMP as a gestational age estimator overestimated the proportion of weight equal to or higher than 2,500g among preterm infants, which does not seem compatible with the expected distribution for this group. LMP favored "correction" of prematurity for the parameters that are comparable to those of primary data studies, though the distortions we found between gestational age and birth weigh may indicate that there are still problems with this estimator.

Information Systems; Birth Certificates;

Gestational Age; Premature Infant; Child Health

\section{Resumen}

La prevalencia de nacimiento pretérmino está presentando una tendencia creciente en varios países, incluso en los desarrollados. Estudios en Brasil reflejan que el Sistema de Información sobre Nacidos Vivos (SINASC), hasta 2010, subestimaba la prevalencia de nacimientos pretérmino, cuando se compara con los estudios basados en datos primarios. A partir de 2011, la edad gestacional al nacer ha sido calculada en el SINASC, cuando se encontraba disponible, mediante la fecha de la última menstruación (DUM). El objetivo fue evaluar la precisión en la determinación de la edad gestacional generada por los DUM, comparándola con la de otros estimadores, y correlacionándola con el peso al nacer. Se trata de un estudio de base poblacional con datos del SINASC, disponibles en el Departamiento de Informática del Sistema Único de Salud entre 2011 y 2015. Las definiciones de prematuridad, bajo peso y asfixia al nacer fueron aquellas determinadas en la literatura. La adecuación del peso al nacer con la edad gestacional se calculó basándose en las curvas de Fenton e Intergrowth-21. Se compararon las medias de peso por la presencia o no de prematuridad. La estimación de la edade gestacional se realizó mediante DUM en un $58,5 \%$, y un $41,5 \%$ utilizaron otro método. Se descubrió que la proporción de prematuridad fue de un $12 \%$ en el grupo DUM y un 8,4\% en el grupo otro método, ya que el bajo peso al nacer fue un $6,5 \%$ y 8,4\%, respectivamente. La media de peso de los prematuros en el grupo DUM fue mayor. El uso de la DUM, como estimador de la edad gestacional, sobreestimó la proporción de peso mayor o igual a $2.500 \mathrm{~g}$ en los prematuros, lo que no parece compatible con la distribución esperada para esta franja. La DUM favoreció la "corrección" de la prematuridad en relación con los parámetros comparables a los de estudios con datos primarios, a pesar de que las distorsiones encontradas entre edad gestacional y peso al nacer puedan indicar que todavía existen problemas con este estimador.

Sistemas de Información; Certificado de Nacimiento; Edad Gestacional; Recién Nacido Prematuro; Salud del Niño
Recebido em 21/Mai/2018

Versão final reapresentada em 27/Nov/2018 Aprovado em 10/Dez/2018 